\title{
Phylogenetic analysis reveals wide distribution of globin X
}

\author{
Jasmin Dröge and Wojciech Makałowski
}

\begin{abstract}
The vertebrate globin gene repertoire consists of seven members that differ in terms of structure, function and phyletic distribution. While hemoglobin, myoglobin, cytoglobin, and neuroglobin are present in almost all gnathostomes examined so far, other globin genes, like globin $X$, are much more restricted in their phyletic distribution. Till today, globin $X$ has only been found in teleost fish and Xenopus. Here, we report that globin $X$ is also present in the genomes of the sea lamprey, ghost shark and reptiles. Moreover, the identification of orthologs of globin X in crustacean, insects, platyhelminthes, and hemichordates confirms its ancient origin.
\end{abstract}

\section{Findings}

Globins are small heme proteins that bind various external ligands, such as oxygen and nitric oxide, and they are found in all kingdoms of life [1]. Best known are hemoglobin $(\mathrm{Hb})$ and myoglobin $(\mathrm{Mb})$, which have been studied for a long time to understand relationships between protein function and structure. In the past ten years, several new members of the globin gene family were added to the vertebrate's globin gene repertoire. Neuroglobin $(\mathrm{Ngb})$ and cytoglobin $(\mathrm{Cygb})$ are highly conserved and present in all gnathostomes examined till today [2-4]. Other novel globin genes are much more restricted in their phyletic distributions, e.g. globin Y (GbY) has been found in Xenopus, lizards, and platypus while globin E (GbE) is only expressed in the eyes of birds [5-7]. Finally, globin X (GbX) seems to be restricted to the genomes of teleost fish and Xenopus [5,8]. The physiological functions of these novel globin proteins are unknown, although a species-specific regulation of GbX under hypoxic conditions has been observed $[9,10]$. Here, we show that the GbX gene is not just restricted to teleost fish and Хепориs genomes, but is also present in the genomes of sea lamprey, ghost shark, and reptiles. Additionally, several invertebrate orthologs of GbX were detected in crustacean, insects, platyhelminthes, and hemichordates, confirming its ancient origin.

The BLAST algorithms [11] were employed to search the non-redundant protein database of NCBI, the

\footnotetext{
* Correspondence: wojmak@uni-muenster.de

Institute of Bioinformatics, Faculty of Medicine, University of Muenster, Niels Stensen Str. 14, 48149 Muenster, Germany
}

sequence databases at the GenBank and the genomic sequences of the sea lamprey, the ghost shark, the Burmese python, and the green anole lizard for homologous globin proteins. Corresponding nucleotide sequences were extracted from the genomes and translation start sites and splice sites were predicted using GENSCAN and GeneNet2, respectively, and manually adjusted [12-14]. The ExPASy Molecular Biology Server at the Swiss Institute of Bioinformatics was used for the translation of the putative CDS (http://www.expasy.org). A table with accession numbers of sequences used in the consecutive analyses and a table with CDS sequences of the newly annotated genes are provided in the supplementary materials [see Additional file 1]. The derived sequences were initially aligned using the MUSCLE program and manually refined [15]. The alignment is provided in the supplementary materials [see Additional file 2]. Phylogenetic trees were reconstructed using maximum likelihood and Bayesian approaches. The program package RAxML 7.2.8 [16,17] was employed for maximum likelihood analyses using the rapid bootstrapping algorithm with 1,000 bootstrap replications. Bayesian analyses were conducted in MrBayes 3.1.2 [18,19] using default priors. Two simultaneous Metropolis-coupled Markov chain Monte Carlo samplings were performed with one cold and three heated chains that were run for 5,000,000 generations. The trees were sampled every $100^{\text {th }}$ generation and the 'burn in' was set to $25 \%$. For the calculation of the phylogenetic trees, the CIPRES portal was used (CIPRES Web Portal V3.1) [20]. Phylogenetic analyses were based 
on the WAG [21] model of amino acid evolution assuming gamma distribution of substitution rates, as suggested by analysis of the alignment with ProtTest3 [22]. The phylogenetic trees were visualized with iTOL [23].

Similarity searches for putative GbX proteins in the $\mathrm{nr}$ protein database of NCBI using GbX of zebrafish as query [GenPept: NP_001012261] resulted in the identification of several homologous invertebrate globin proteins and a partial GbX sequence of the green anole lizard [GenPept: XP_003228427]. A tBLASTn search against several vertebrate genomes revealed that GbX is not restricted to teleost fish and amphibians. The GbX gene is also present in the genomes of green anole lizard, Burmese python, ghost shark, and sea lamprey. While teleost fish possess only one copy of the GbX gene, $X$. tropicalis may indeed contain two GbX genes [5]. For lizard and python we obtained sequences that each correspond to a single GbX gene. Interestingly, ghost shark and sea lamprey may possess two copies of the GbX gene. The coding regions of fish and amphibian GbX are distributed on five exons. The introns are located at positions B12.2 (i.e. between the second and third base of the $12^{\text {th }}$ codon in globin helix B), G7.0, E10.2 and H10.0 [8]. The putative lizard and python GbX genes consist of four coding exons and were found on chrUn_GL343635 and on several contigs (contig24997472, contig25376860, contig25192522, contig25757037), respectively. We couldn't locate the first coding exon of fish and Xenopus GbX, comprising the $\mathrm{N}$-terminal extension and some part of the globin domain (on average the first 57 amino acids), completely in the lizard and partially in the python genome. Moreover, in python the last coding exon comprising the $\mathrm{C}$-terminal extension of the protein seems to be absent. Interestingly, we were able to identify the first coding exon in the previous assembly of the lizard genome (anoCar1.0). Thus, it is most likely that complete first and last coding exons couldn't be found due to missing sequence data and/or due to miss-assembly. The potential CDSs of lizard and python GbX translate into peptides of 200 and 129 amino acids, respectively. The putative ghost shark and sea lamprey GbX genes consist of five coding exons and are distributed on several scaffolds (AAVX01210948, AAVX0160156, AAVX01601570, AAVX01646770, AAVX01022872, AAVX01385372, AAVX01173359, AAVX01250480) and contigs (contig19308, contig21159, contig11157, contig27503), respectively. The putative cDNAs of ghost shark and sea lamprey GbX translate into peptides of 193 and 222 amino acids, respectively. Interestingly, in both species we found partial sequences of a putative second GbX gene (GbX-2). The partial GbX-2 genes of lamprey and shark reside on contig20413, contig362 and on AAVX01509327, AAVX01477794, respectively. For the sea lamprey coding exons two to four were identified that translate into a peptide of 103 amino acids that is $71 \%$ identical and (based on BLOSUM62) 88\% similar to the other copy of GbX (GbX-1). For the ghost shark only coding exons two and three were identified that translate into a peptide of 75 amino acids. The GbX-2 peptide of the ghost shark is $67 \%$ identical and $83 \%$ similar to GbX1. Since large parts of the putative GbX-2 genes couldn't be found in the genomes, those sequences might represent pseudo-genes. The key residues important in oxygen binding, like the proximal and distal histidines in the Eand F-helix (HisE7, HisF8) and the phenylalanine in the $\mathrm{CD}$ region (PheCD1) are strictly conserved in all new annotated GbX proteins.

The newly found vertebrate GbX proteins, the putative orthologous globin proteins of the lancelet [24] as well as several homologous invertebrate globin proteins were added to the alignment of vertebrate GbX and Ngb proteins. We refrained from including the GbX-2 proteins of the ghost shark and sea lamprey because of the incompleteness of the data. The sequences of pea aphid and Daphnia pulex globin proteins were corrected based on available EST sequences (GL350495, FF335385, FE368658, FE407975 and FE407974). Various phylogenetic trees were constructed employing the maximum likelihood and Bayesian algorithms. Because of the high divergence of non-metazoan globin proteins and in order to identify orthologous relationships between GbX and invertebrate globin proteins, the closest vertebrate relative of GbX, namely Ngb, was treated as an outgroup (DreNgb, XtrNgb). As expected, the new annotated GbX proteins from sea lamprey, ghost shark and reptiles group with GbX from teleost fish and Xenopus with high bootstrap support (Figure 1). However, the clustering of the $\mathrm{GbX}$ proteins is not in accordance with the species tree. For example, in our phylogenetic trees GbX from sea lamprey is closer related to GbX of reptiles than to GbX of the ghost shark. The discrepancy of gene and species tree may be caused by the overall comparable similarity of the GbX proteins which ranges from $70 \%$ to $88 \%$ (based on BLOSUM62). However, under the assumption that the gene tree is correct, we would assume a scenario in which the last common ancestor of vertebrates possessed multiple copies of GbX and in which several losses of GbX genes occurred in the evolution of vertebrates. In this scenario the GbX proteins of e.g. teleost fish and reptiles would rather be paralogs than orthologs. Analyses of additional vertebrate genomes will be necessary to resolve this issue. Interestingly, in all phylogenetic reconstructions, the vertebrate GbX proteins consistently group with putative globin proteins of the pea aphid (ApiGb1), human body louse (PhucoGbD), Daphnia pulex (DpuGb), two Schistosoma species (SjaGb, SmaGb), and the acorn worm (Saccoglossus kowalevskii) (SkoGb1-4). This branching was supported 


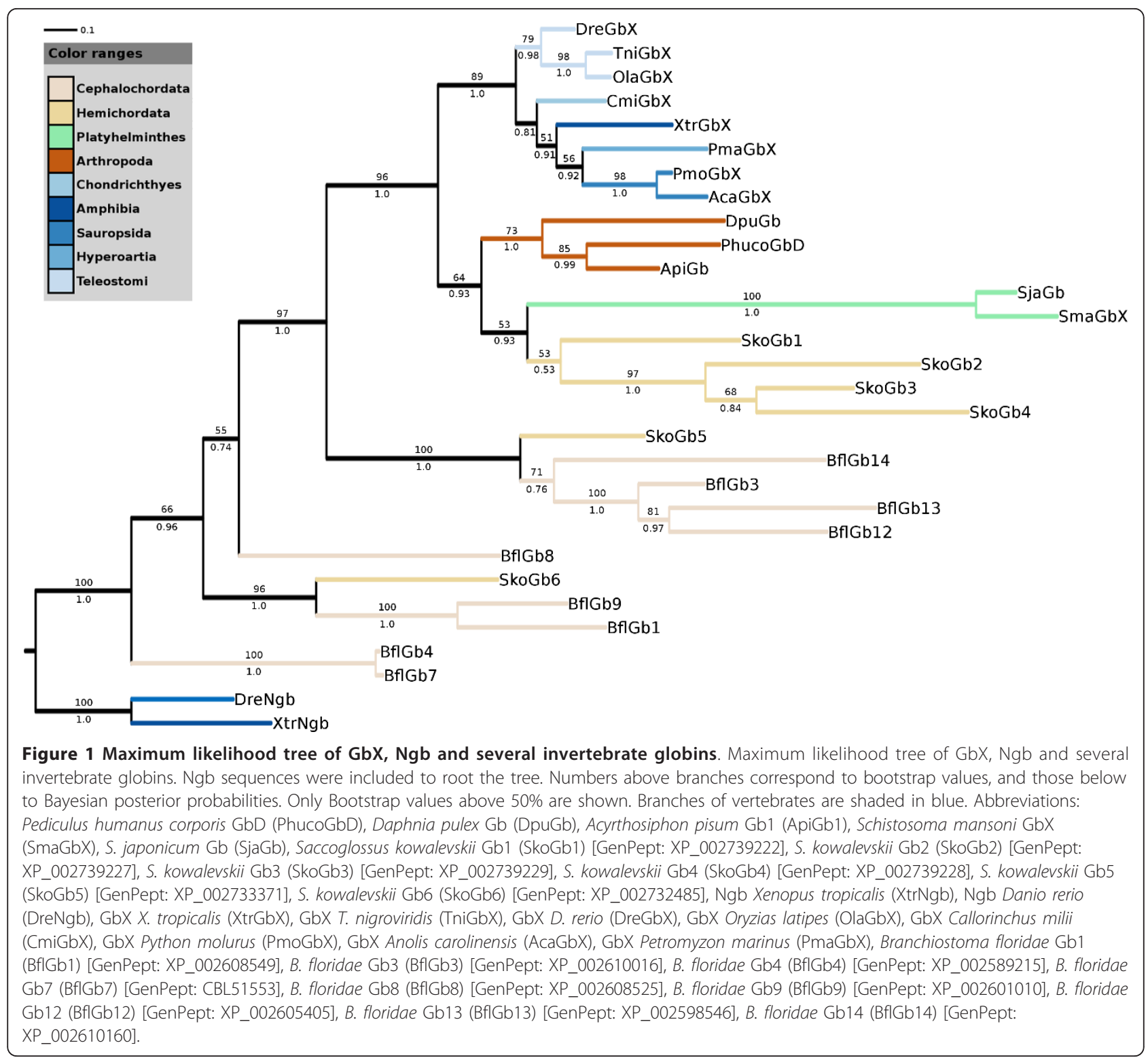

by bootstrap support up to $96 \%$ depending on the number of analyzed species and the kind of analysis. Support for orthology of these globin proteins was further obtained by analyzing the organization at the genomic level. Within teleost fish and Xenopus, the genomic region containing $\mathrm{GbX}$ is conserved in gene order and arrangement. The GbX gene of the zebrafish resides on chromosome 17 between the genes for signal recognition particle 14 (srp14) and pleckstrin homology domain-containing family G, member 3 (plekgh3) [5,24]. The putative orthologous globin proteins of the acorn worm (SkoGb1-4) lie next to each other on one genomic scaffold and thus may have arisen by duplications of an ancestral GbX gene. Interestingly, they are close to the putative ortholog of srp14 [see Additional file 3]. Thus, we propose that these globins are orthologs of vertebrate GbX. Further phylogenetic interpretation of the tree suggests that the monophyletic clade, comprising the putative orthologous globin proteins of the lancelet (BflGb3, BflGb12-14) and an additional putative globin from the acorn worm (SkoGb5), contains paralogs of vertebrate GbX. The SkoGb5 gene of the acorn worm resides in a similar genomic neighborhood as GbX of the analyzed euteleostei fish species. While the direct neighbors of vertebrate $\mathrm{GbX}$ are not detectable on this scaffold, the homologs of gremlin 1 (grem1) and dnaj (HSP40) homolog subfamily $\mathrm{C}$ member 17 (dnajc17), both located downstream of euteleostei fish GbX, reside in close proximity of SkoGb5. Hence, it is likely that this genomic organization arose by a duplication of the region comprising the ancestral GbX 
gene. Thus, we propose that the globin SkoGb5 of the acorn worm as well as the amphioxus globins (BflGb3, BflGb12-14) are paralogous to vertebrate GbX and that, most probably, the lancelet and higher vertebrates have each lost one copy of GbX.

Our phylogenetic analysis supports the scenario that the GbX gene is a product of a duplication event that predates the divergence between protostomes and deuterostomes $[8,24]$. As reported before, we were not able to detect GbX in the genomes of mammals and birds. Thus, it seems that GbX was lost twice in vertebrate evolution. Interestingly, the vertebrate lineages that possess a GbX gene represent ectothermic animals while those who lost the GbX gene are endotherms. This distribution may give some hints towards GbX function. Moreover, it will be interesting to see if the ectothermic crocodiles, which are a sister group of birds, own a GbX gene. Crocodiles possess a four-chambered heart which is a characteristic of endotherms in which higher metabolic rates would select for the perfect separation of blood. This led to the hypothesis that the ancestors of crocodilians and birds were endothermic [25]. It will be intriguing to see if GbX was lost in the lineage leading to birds or if was already lost in stem archosaurs.

\section{Reviewers' comments}

\section{Reviewer's report 1: Gáspár Jékely, Max Planck Institute} for Developmental Biology, Tübingen, Germany

This paper describes novel globin $\mathrm{X}$ genes from several vertebrates and invertebrates. The classification of these genes as globin $\mathrm{X}$ is confirmed by molecular phylogeny.

Since some nodes in the phylogeny are not well resolved, and there are some groupings inconsistent with the species tree (e.g. the sequences from the deuterostome Saccoglossus grouping with protostome ones), it would be worth showing the alignments as a separate figure. In the alignment members of other globin families should also be included. This could reveal if the globin X group has some shared sequence signatures, distinguishing them from other globins.

The authors should also comment on the putative amphioxus globin X orthologues described in ref 24. The tree in Figure 1 indicates that the amphioxus genes (BflGb3,12,13,14) represent a distinct class, maybe originating at a separate duplication event, predating bilaterian radiation. BflGb6 is missing from the tree.

\section{Author's response}

Thank you for the insightful comments. Globin sequences are difficult to align, indeed. We agree that in such cases it is useful to provide the alignment, which is now available in phylip format as an additional file 2 . The amphioxus globin BflGb6 was excluded from the analysis because no globin domain was detected using the normal mode of SMART. The sequence may be mis-annotated since a part of the globin domain is absent in the current version. Other members of the vertebrate globin gene family were not added to the alignment to improve readability of the tree. Moreover, globin X sequences share only little sequence similarity with other vertebrate globin types. We agree that the amphioxus genes (BflGb3, $12,13,14)$ represent a distinct class derived from a separate duplication event predating bilaterian radiation.

\section{Reviewer's report 2: Arcady Mushegian, Stowers Institute} for Medical Research, United States of America

This is an interesting observation of broader-thenexpected distribution of globin $\mathrm{X}$ among metazoa, and of the possible connection between globin $\mathrm{X}$ and ectothermy. It is suitable for publication as a Discovery Note, with perhaps one clarification. How do we know what was the ingroup and what was the outgroup, i.e., what are $\mathrm{X}$ and what are non-X? Apparently, these calls were made before tree inference, but in principle, the ultimate orthology assignment can be only done by examination of the phylogenetic tree. I suspect that the answer is that globin $\mathrm{X}$ sequences were much closer to each other than to any other globins, and therefore the extent of the family could be defined without tree mapping - but this was not stated in the manuscript.

\section{Author's response}

Thank you for your comment that gives us the opportunity to clarify our methodology. The analysis was conducted without prior expectations. A BLAST search was carried out using vertebrate globin $\mathrm{X}$ as query to search for similar sequences in invertebrate species. Subsequently, phylogenetic trees were computed to identify relationships between sequences. Neuroglobin was chosen as an outgroup, because it is assumed that neuroglobin emerged before the split of protostomier and deuterostomier. Moreover, globin X is closer related to neuroglobin than to any other vertebrate globin type with identity values ranging from $26 \%$ to $34.6 \%$.

\section{Additional material}

Additional file 1: Tables of used sequences. A table of used sequences, along with accession number and gi-number, and a table of CDS sequences of the new annotated sequences are provided in this file. Additional file 2: Amino acid sequence alignment of GbX from zebrafish, medaka, Tetraodon and Xenopus, Ngb from zebrafish and Xenopus, the newly annotated vertebrate GbX proteins, the putative orthologous globin proteins of the lancelet and several homologous invertebrate globin proteins. The alignment is provided in interleaved phylip format.

Additional file 3: Schematic comparison of the gene neighborhood of GbX from medaka chromosome 22 to scaffolds 14411 and 38908 of the S. kowalevskii genome and to scaffold 29 of the B. floridae genome. Arrows indicate the location of the genes (right handed arrow = plus strand, left handed arrow = minus strand). Genes drawn in the same color are homologs. Genes drawn in light grey are not

homologous to other genes in the same chromosomal position of the 
other species. Dots indicate that shown genes are separated by more than one gene.

\section{Acknowledgements and Funding}

This work has been supported by the Institute of Bioinformatics funds.

\section{Authors' contributions}

JD designed the study, carried out the bioinformatic analyses and drafted the manuscript. WM conceived of the study, participated in its design and coordination and helped to draft the manuscript. All authors read and approved the final manuscript.

\section{Competing interests}

The authors declare that they have no competing interests.

Received: 26 August 2011 Accepted: 17 October 2011

Published: 17 October 2011

\section{References}

1. Hardison RC: A brief history of hemoglobins: plant, animal, protist, and bacteria. Proc Natl Acad Sci USA 1996, 93:5675-5679.

2. Burmester $T$, Weich $B$, Reinhardt $S$, Hankeln T: A vertebrate globin expressed in the brain. Nature 2000, 407:520-523.

3. Burmester T, Ebner B, Weich B, Hankeln T: Cytoglobin: a novel globin type ubiquitously expressed in vertebrate tissues. Mol Biol Evol 2002. 19:416-421.

4. Trent JT, Hargrove MS: A ubiquitously expressed human hexacoordinate hemoglobin. J Biol Chem 2002, 277:19538-19545.

5. Fuchs $C$, Burmester $T$, Hankeln T: The amphibian globin gene repertoire as revealed by the Xenopus genome. Cytogenet Genome Res 2006, 112:296-306.

6. Patel VS, Cooper SJ, Deakin JE, Fulton B, Graves T, Warren WC, Wilson RK, Graves JA: Platypus globin genes and flanking loci suggest a new insertional model for beta-globin evolution in birds and mammals. BMC Biol 2008, 6:34.

7. Kugelstadt D, Haberkamp M, Hankeln T, Burmester T: Neuroglobin, cytoglobin, and a novel, eye-specific globin from chicken. Biochem Biophys Res Commun 2004, 325:719-725.

8. Roesner A, Fuchs C, Hankeln T, Burmester T: A globin gene of ancient evolutionary origin in lower vertebrates: evidence for two distinct globin families in animals. Mol Biol Evol 2005, 22:12-20.

9. Roesner A, Hankeln T, Burmester T: Hypoxia induces a complex response of globin expression in zebrafish (Danio rerio). J Exp Biol 2006, 209:2129-2137.

10. Wawrowski A, Gerlach F, Hankeln T, Burmester T: Changes of globin expression in the Japanese medaka (Oryzias latipes) in response to acute and chronic hypoxia. J Comp Physiol B 2011, 181:199-208.

11. Altschul SF, Gish W, Miller W, Myers EW, Lipman DJ: Basic local alignment search tool. J Mol Biol 1990, 215:403-410.

12. Brunak S, Engelbrecht J, Knudsen S: Prediction of human mRNA donor and acceptor sites from the DNA sequence. J Mol Biol 1991, 220:49-65.

13. Hebsgaard SM, Korning PG, Tolstrup N, Engelbrecht J, Rouze P, Brunak S: Splice site prediction in Arabidopsis thaliana pre-mRNA by combining local and global sequence information. Nucleic Acids Res 1996 24:3439-3452

14. Burge CB, Karlin S: Finding the genes in genomic DNA. Curr Opin Struct Biol 1998, 8:346-354.

15. Edgar RC: MUSCLE: multiple sequence alignment with high accuracy and high throughput. Nucleic Acids Res 2004, 32:1792-1797.

16. Stamatakis A: RAxML-VI-HPC: maximum likelihood-based phylogenetic analyses with thousands of taxa and mixed models. Bioinformatics 2006 22:2688-2690.

17. Stamatakis A, Hoover P, Rougemont J: A rapid bootstrap algorithm for the RAxML Web servers. Syst Biol 2008, 57:758-771.

18. Ronquist F, Huelsenbeck JP: MrBayes 3: Bayesian phylogenetic inference under mixed models. Bioinformatics 2003, 19:1572-1574.

19. Huelsenbeck JP, Ronquist F: MRBAYES: Bayesian inference of phylogenetic trees. Bioinformatics 2001, 17:754-755.
20. Miller MA, Holder MT, Vos R, Midford PE, Liebowitz T, Chan L, Hoover P, Warnow T: The CIPRES Portals. CIPES. 2009 [http://www.phylo.org/ sub_sections/portal].

21. Whelan S, Goldman N: A general empirical model of protein evolution derived from multiple protein families using a maximum-likelihood approach. Mol Biol Evol 2001, 18:691-699.

22. Abascal F, Zardoya R, Posada D: ProtTest: selection of best-fit models of protein evolution. Bioinformatics 2005, 21:2104-2105.

23. Letunic I, Bork P: Interactive Tree Of Life (iTOL): an online tool for phylogenetic tree display and annotation. Bioinformatics 2007, 23:127-128.

24. Ebner B, Panopoulou G, Vinogradov SN, Kiger L, Marden MC, Burmester T, Hankeln T: The globin gene family of the cephalochordate amphioxus: implications for chordate globin evolution. BMC Evol Biol 2010, 10:370.

25. Seymour RS, Bennett-Stamper CL, Johnston SD, Carrier DR, Grigg GC: Evidence for endothermic ancestors of crocodiles at the stem of archosaur evolution. Physiol Biochem Zool 2004, 77:1051-1067.

doi:10.1186/1745-6150-6-54

Cite this article as: Dröge and Makałowski: Phylogenetic analysis reveals wide distribution of globin X. Biology Direct 2011 6:54.

\section{Submit your next manuscript to BioMed Central and take full advantage of:}

- Convenient online submission

- Thorough peer review

- No space constraints or color figure charges

- Immediate publication on acceptance

- Inclusion in PubMed, CAS, Scopus and Google Scholar

- Research which is freely available for redistribution

Submit your manuscript at www.biomedcentral.com/submit 